INTERNATIONAL JOURNAL OF RESEARCHES IN BIOSCIENCES, AGRICULTURE AND TECHNOLOGY (C) VISHWASHANTI MULTIPURPOSE SOCIETY (Global Peace Multipurpose Society) R. No. MH-659/13(N) www.vmsindia.org

\title{
ENVIRONMENTAL ACCEPTABLE AND CLASSICAL SYNTHESIS OF 7-(4-ARYLIDENE BENZYLIDENE ACETOPHENONE) SUBSTITUTED PHENOTHIAZINE AND THEIR ANTIBACTERIAL ACTIVITY
}

\author{
M. N. Narule ${ }^{1}$ and S. B. Rewatkar ${ }^{2}$ \\ ${ }^{1}$ Vidya Vikas Art, Commerce and Science College, Samudrapur, Dist-Wardha, Maharashtra, INDIA. \\ ${ }^{2}$ Mohsinbhai Zaweri College, Desaiganj(Wadsa) Dist - Gadchiroli Maharashtra, INDIA. \\ sbrewatkar@gmail.com
}

\begin{abstract}
:
Synthesis of 4-amino benzylidene acetophenone 1 was prepared by condensing acetophenone with 4-amino aldehydes. 4-amino benzylidene acetophenone react with 4-hydroxy aldehyde to give 4-Arylidene (4-hydroxy phenyl) benzylidene acetophenone 2 combine with substituted anilines gives 4-Arylidene phenyl (4-amino benz) benzylidene acetophenone 3. 4-Arylidene phenyl (4-amino benz) benzylidene acetophenone undergo cyclization with sulpher and iodine gives 7-(4Arylidene benzylidene acetophenones) substituted phenothiazine $4 \mathrm{a}-\mathrm{j}$. All these reaction are carried out in borosil beaker under microwave irradiation in microwave oven. The structural elucidations of these compounds were done on the basis of chemical and structural data. The antibacterial activity of these compounds have also been screened and found to be effective against gram +ve and gram -ve bacteria.

Keyw ords; Acetophenones, phenothiazine, aniline, microwave.
\end{abstract}

\section{Introduction:}

The chemistry of benzylideneacetophenone has generated intensive scientific interest due to their biological and applications. Benzylidene acetophenones and their derivatives possess some interesting biological properties such as antibacterial $^{1} \quad$ antifungal $^{2}$, insecticidal ${ }^{3}$, anaesthetic, ant i- inflammatory, analgesic, ulcerogenic ${ }^{4-5}$ etc. Benzylideneacetophenones play an ecological role in nature, in relation to plant colour. These brightly yellow colored compounds are found in many plant organs, but most conspicuously in flowers. Benzylidene acetophenones contain reactive keto ethylenic (enone) group. The presence of enone fraction in the benzylidene acetophenones molecule confers antibiotic activity (bacteriostatics / bactericidal) upon it. This property is enhanced when substitution is made at the a-(nitro and bromo) and $\beta$-(bromo and hydroxylic) position. Some substituted benzylidene acetophenones and their derivatives, including some of their heterocyclic analogues, have been reported to possess some interesting biological properties, 6-9 which are detrimental to the growth of microbes, tubercle bacilli, malarial parasites, acrus, Schistosoma, and intestinal worms, Some of the compounds are claimed to be toxic to animals and insects and are also reported to exhibit inhibitory action on several enzymes, fungi, and herbaceous plants. Schiff's bases derivatives possess wide range of pharmacological activities like antioxidant ${ }^{10}$, antiinvasive $11-13$, antivitral, antipyretic, antiinflammatory, antidepressant, and blood pressure lowering 14-16 Phenothiazine derivatives are an important class of five-membered heterocycles associated with biological activities like antibacterial, antiviral , anti - inflammatory, antifungal, anti tuberculosis, antibiotic, antileprous ${ }^{17}$ psychotherapy 18 anabolic, analgesics agents, agricultural fungicide and in acutely ill HIV-infected patients ${ }^{19-23}$. Microwave-induced organic Reaction Enhancement (MORE).is used for carrying out chemical transformations ${ }^{24}$. The microwave assisted organic reactions are more safe and an environmentally friendly with enhanced purity and yields ${ }^{25}$ of products. Shorter reaction time periods and higher yields render the microwave method superior to the classical method.

\section{Result and Discussion:}

In view of the above mention pharmacological activities of benzylidene acetophenone, Schiff's bases and Phenothiazine a number of the 7-(4-Arylidene benzylidene acetaophenone) substituted phenothiazine derivatives have been synthesized which containing above moieties

The reaction sequence leading to the formation of desired heterocyclic compounds are outlined in Scheme-I. The starting material 4amino benzylidene acetophenone $\mathbf{1}$ was prepared by the reaction of 4-amino benzaldehyde with acetophenone in presence of $40 \% \mathrm{NaOH}$ which on treatment with 4-hydroxy aldehyde reacts to give 4Arylidene (4-hydroxy phenyl) benzylidene acetophenone $\mathbf{2}$ combine with substituted anilines gives 4-Arylidene phenyl (4-amino benz) benzylidene acetophenone 3. 4-Arylidene phenyl (4amino benz) benzylidene acetophenone undergo Structural assignment of synthesized compounds is based on the spectral data. IR spectral bands of all the compounds indicates peak at $690-840 \mathrm{~cm}^{-1}$ (substituted phenyl) and number of peaks at 1020$1340,1400-1500,3050$ and 3300-3400 for C-N stretching $\mathrm{C}=\mathrm{C}$, aromatic Ar- $\mathrm{H}$ stretching and $\mathrm{N}-\mathrm{H}$ stretching respectively. A characteristic peak at 1600-1660 $\mathrm{cm}^{-1}$ indicates the presence of $\mathrm{C}=\mathrm{N}$ band. The PMR spectrum indicates singlets for $\mathrm{N}-\mathrm{H}$, phenothiazine. The benzenoid protons appeared in the range of $6.40-7.60$. The $\mathrm{m} / \mathrm{z}$ molecular ion peak for 2, 34 appeared at 333, 363.06and 375.0 respectively. A reaction should be conducted under solvent-free conditions with minimal or no side product formation and with utmost atom economy. In classical method the yield is lower as compared to microwave irradiation. Microwave irradiation 


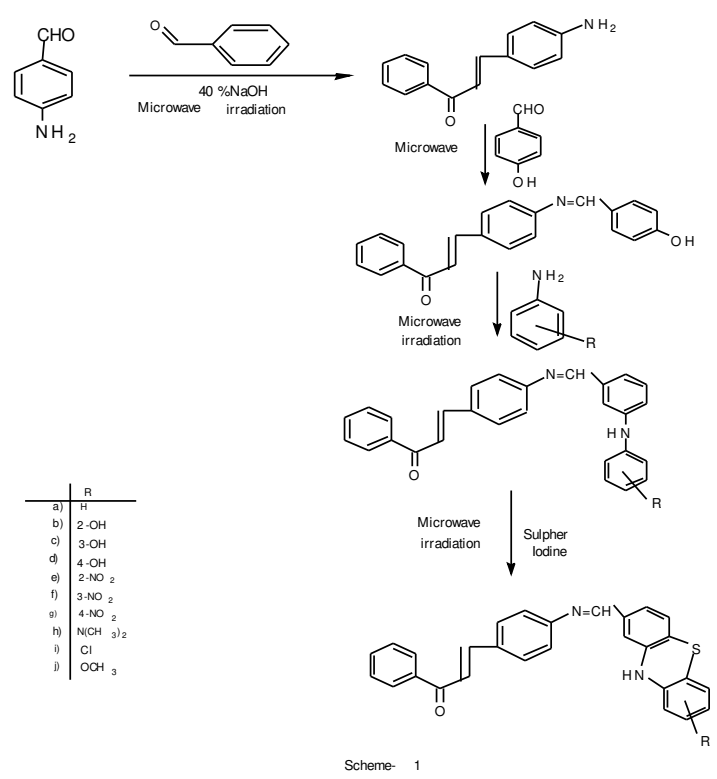

facilitates the polarization of the molecule under irradiation causing rapid reaction. A comparative study in terms of yield and reaction period is shown in Table-II

\section{Antimicrobial activity}

The compounds $\mathbf{4 a - j}$ were screened for their antibacterial activity against Bacillus subtilis, staphylococcus aureus and Escherichia coil and antifungal activity against Candida albicans and Aspergillus nigar by filter paper disc techniquc ${ }^{13}$. Standard antibacterial Streptomycin and antifungal Griscofulvin were also tested under similar conditions for comparison. Results are presented in Table I

Synthesis of 4 from 3

By microwave irradiation method:

A) Solid phase MWI - A solution of $\mathbf{3}(0.01 \mathrm{~mol})$, iodine and sulphur $(0.01 \mathrm{~mol})$ in ethanol $(2 \mathrm{ml})$ was taken in a $100 \mathrm{ml}$ borosil flask and to this $\mathrm{KOH}(1 \mathrm{~g})$ and basic alumina $(3 \mathrm{~g})$ was added. The reaction mixture was thoroughly mixed, dried in air and irradiated inside a microwave oven for 2$3 \mathrm{~min}$. at power level(700W), the reaction mixture was cooled and extracted with ethanol $(3 \times 10 \mathrm{ml})$.The resultant solid was recrystallized using aqueous ethanol.

B) Solution phase MWI -Equimolar quantities of 3 iodine and sulphur $(0.01 \mathrm{~mol})$ in ethanol $(30 \mathrm{ml})$ were taken in a $100 \mathrm{ml}$ borosil flask fitted with a funnel as a loose top. The reaction mixture was irradiated in a microwave oven for 5-6 min. at $20 \%$ power level (300W) with short interruption of $20 \mathrm{sec}$, to avoid the excessive evaporation of the solvent. This protocol was repeated in overall heating time. On completion of the reaction (TLC) the reaction mixture was cooled and acidified with dil $\mathrm{HCl}$. The product 4 separated was filtered, washed with cold water, dried and recrystallized from ethanol.

\section{Experimental}

Melting points were determined and are uncorrected. Purity of the compounds was checked on TLC using iodine vapor as visualizing agent. The IR spectra were run in $\mathrm{KBr}$ on a Perkins - Elmer infrared spectrophotometer. ${ }^{1} \mathrm{H}$ NMR spectra on Bucker $\mathrm{AC}-300 \mathrm{~F}(300 \mathrm{~Hz})$ NMR spectrometer using DMSO as a solvent using tetra methyl silence as internal standard.

\section{4-amino benzylidene acetophenone 1}

Acetophenone $(0.01 \mathrm{~mol})$ and 4-amino benzaldehyde $(0.01 \mathrm{~mol})$ was dissolved in $100 \mathrm{ml}$ ethanol. To this solution, $\mathrm{NaOH}(40 \%, 10 \mathrm{ml})$ was added drop wise with constant stirring at room temperature till a dark yellow mass was obtained. The reaction mixture was kept 7-8 hr and acidified with dil $\mathrm{HCl}$. The solid obtained was washed with cold water. It was filtered and dried. It was crystallized from ethanol. Yield $85 \%$ M.P 1530

4-Arylidene (4-hydroxy phenyl) benzylidene acetophenones 2

4-amino benzylidene acetophenone (0.05mole) and an 4-hydroxy aldehyde amine (0.05mole) in absolute ethanol (50ml) was heated under refluxed for $6 \mathrm{hr}$ in water bath and then wash with acidified water to remove inorganic materials, then it was filtered off to obtain 2and crystallized from ethanol.

\section{4-Arylidene phenyl (4-amino Benz) benzylidene acetophenones 3}

4-Arylidene (4-hydroxy phenyl) benzylidene acetophenone 2(0.05mole with different aromatic primary amine $(0.05 \mathrm{~mole})$ in absolute ethanol $(50 \mathrm{ml})$ was heated under reflux in the presence of anhydrous. $\mathrm{ZnCl}_{2}(0.5 \mathrm{~g})$ for $6 \mathrm{hr}$. on a water bath. On cooling, a solid mass separated out which was wash with acidified water to remove inorganic materials, then it was filtered off to obtain 3and crystallized from ethanol.

\section{7-(4-Arylidene benzylidene acetophenone)} substituted phenothiazine 4

A mixture of 4-Arylidene phenyl (4-amino Benz) benzylidene acetophenone 3 (0.01mole) sulpher ( 0.1 mole) and Iodine $(0.5 \mathrm{~g})$ was rapidly heated at $120^{\circ} \mathrm{c}$ in an oil bath for $2 \mathrm{hr}$. The hot melt was rapidly poured in to a mortar and crushed to a fine powder, to give 4 It was washed with water dried and crystallized from ethanol.

7-(4-Arylidene benzylidene acetophenone) substituted phenothiazine 4a

Yield $71 \%$, M.P. $216^{\circ} \mathrm{C}$ : IR (KBr) ; 3570 (NHpyrrole) , 3422 (NH-phenothiazine) , 3324 (NHpyrazole) , $1635(\mathrm{ArH}), 1445(\mathrm{C}=\mathrm{N}), 817(\mathrm{C}-\mathrm{N})$, 740 (C-S) ; ${ }^{1} \mathrm{HNMR}$ (300 MHz DMSO) 88.28 (1H,s,NH-pyrrole) $\quad, \quad 7.8 \quad(1 \mathrm{H}, \mathrm{s}, \mathrm{N}-\mathrm{H}-$ phenothiazine), $7.28 \quad(1 \mathrm{H}, \mathrm{s}, \mathrm{NH}$-pyrazole $)$ $6.8(5 \mathrm{H}, \mathrm{m}, \mathrm{ArH})$

7-(4-Arylidene benzylidene acetophenone) substituted phenothiazine 4b. Yield $67 \%$, M.P. $207^{\circ} \mathrm{C}$ : IR(KBr); $3532 \quad$ (NH-pyrrole), 34269(NH-phenothiazine), 3344 (NH-pyrazole), 1630(ArH), $1443(\mathrm{C}=\mathrm{N}), \quad 814(\mathrm{C}-\mathrm{N}), \quad 730(\mathrm{C}-\mathrm{S})$; ${ }^{1} \mathrm{HNMR} \quad(300 \mathrm{MHzDMSO}) \quad \delta \quad 8.20 \quad(1 \mathrm{H}, \mathrm{s}, \mathrm{NH}-$ pyrrole), 7.7 (1H,s,N-H-phenothiazine), 7.18 (1H,s, NH-pyrazole) $6.8(5 \mathrm{H}, \mathrm{m}, \mathrm{ArH})$,

7-(4-Arylidene benzylidene acetophenone) substituted phenothiazine $4 \mathrm{c}$. 
Yield 65\%, M.P. $121^{\circ} \mathrm{C}$ : IR(KBr) ; $3570(\mathrm{NH})$, 34229 (NH- phenothiazine), 3324 (NHpyrazole), $1635(\mathrm{ArH}), 1445(\mathrm{C}=\mathrm{N}), 817(\mathrm{C}-\mathrm{N})$, 740(C-S); ${ }^{1} \mathrm{HNMR}$ (300 MHz DMSO) $\delta 8.28$ (1H,s, NH-pyrrole) , 7.8 (1H,s, N-H-phenothiazine) , $7.28(1 \mathrm{H}, \mathrm{s}, \mathrm{NH}$-pyrazole) $6.8(5 \mathrm{H}, \mathrm{m}, \mathrm{ArH})$.

7-(4-Arylidene benzylidene acetophenone) substituted phenothiazine 4d.Yield 58\%, M.P. $210^{\circ} \mathrm{C}$ : IR (KBr) ; 3570 (NH-pyrrole) , 34229 (NH-phenothiazine) , 3324 (NH-pyrazole) ,1635 $(\mathrm{ArH}), 1445(\mathrm{C}=\mathrm{N}), 817(\mathrm{C}-\mathrm{N}), 740(\mathrm{C}-\mathrm{S}) ;{ }^{1} \mathrm{HNMR}$ (300MHz DMSO) $\delta 8.28$ (1H,s, NH-pyrrole) , 7.8 $(1 \mathrm{H}, \mathrm{s}, \mathrm{N}-\mathrm{H}-\mathrm{phenothiazine}) \quad, \quad 7.28 \quad(1 \mathrm{H}, \mathrm{s}, \mathrm{NH}-$ pyrazole) $6.8(5 \mathrm{H}, \mathrm{m}, \mathrm{ArH})$.

7-(4-Arylidene benzylidene acetophenone) substituted phenothiazine 4e (6e).Yield $78 \%$, M.P $194^{\circ} \mathrm{C}$ : $\quad \operatorname{IR}(\mathrm{KBr}) ; \quad 3570(\mathrm{NH}), \quad 34229(\mathrm{NH}-$ phenothiazine), 3324(NH-pyrazole), 1635(ArH), 1445(C=N), $817 \quad(\mathrm{C}-\mathrm{N}) \quad, \quad 740(\mathrm{C}-\mathrm{S})$; ${ }_{1}^{1}$ HNMR $\quad(300 \mathrm{MHz}$ DMSO $) \delta 8.28 \quad(1 \mathrm{H}, \mathrm{s}, \mathrm{NH}-$ pyrrole) ,7.8 (1H,s,N-H-phenothiazine) , 7.28 $(1 \mathrm{H}, \mathrm{s}, \mathrm{NH}$-pyrazole) $6.8(5 \mathrm{H}, \mathrm{m}, \mathrm{ArH})$.

7-(4-Arylidene benzylidene acetophenone) substituted phenothiazine $4 \mathrm{f}$

Yield $68 \%$, M.P. $215^{\circ} \mathrm{C}$ : IR (KBr); $3685(\mathrm{OH})$, 3320 (NH-pyrrole), $1620(\mathrm{ArH}), 1422(\mathrm{C}=\mathrm{N})$, $1320\left(\mathrm{CH}_{3}\right), 1545\left(\mathrm{C}-\mathrm{NO}_{2}\right), 842(\mathrm{C}-\mathrm{N}) ;{ }^{1} \mathrm{HNMR}$

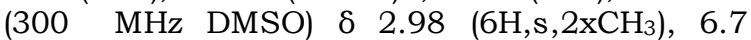
$(5 \mathrm{H}, \mathrm{m}, \mathrm{ArH}) 8.51(1 \mathrm{H}, \mathrm{s}, \mathrm{NH}))$.

7-(4-Arylidene benzylidene acetophenone) substituted phenothiazine $4 \mathrm{~g}$

Yield $64 \%$, M.P. $199^{\circ} \mathrm{C}$ : $\operatorname{IR}(\mathrm{KBr}) ; 3560 \quad(\mathrm{OH})$, $3570(\mathrm{NH}), 1635(\mathrm{ArH}), 1445(\mathrm{C}=\mathrm{N}), 1320\left(\mathrm{CH}_{3}\right)$, 817 (C-N) , 740(C-Cl); ${ }^{1}$ HNMR (300MHzDMSO) $83.21\left(6 \mathrm{H}, \mathrm{s}, 2 \mathrm{xCH}_{3}\right) \quad, 6.8 \quad(5 \mathrm{H}, \mathrm{m}, \mathrm{ArH}), \quad 8.28$ $(1 \mathrm{H}, \mathrm{s}, \mathrm{NH})$.

7-(4-Arylidene benzylidene acetophenone) substituted phenothiazine $4 \mathrm{~h}$.

Yield $81 \%$, M.P. $220^{\circ} \mathrm{C}$ : IR(KBr) ; 3520(NHpyrrole), 34224 (NH-phenothiazine), 3321 (NHpyrazole) , $1635(\mathrm{ArH}), 1445(\mathrm{C}=\mathrm{N}), 817(\mathrm{C}-\mathrm{N})$, 740(C-S); 1HNMR (300 MHz DMSO) $\delta 8.28$ (1H,s,NH-pyrrole) , 7.8 (1H,s, N-H-phenothiazine) , 7.28 (1H,s, NH-pyrazole) $6.8(5 \mathrm{H}, \mathrm{m}, \mathrm{ArH})$.

7-(4-Arylidene benzylidene acetophenone) substituted phenothiazine $4 i$

Yield $65 \%$, M.P. $123^{\circ} \mathrm{C}$ : IR(KBr) ; 3577 (NH- pyrrole), 3424 (NH-phenothiazine) , 3344 (NHpyrazole), 1637(ArH), $1445(\mathrm{C}=\mathrm{N}), 817(\mathrm{C}-\mathrm{N})$, 740(C-S) ; 1HNMR (300MHzDMSO) $\delta 8.28$ (1H,s,NH-pyrrole) , 7.8 (1H,s, N-H-phenothiazine) , $7.28(1 \mathrm{H}, \mathrm{s}, \mathrm{NH}$-pyrazole) $6.8(5 \mathrm{H}, \mathrm{m}, \mathrm{ArH})$.

\section{7-(4-Arylidene benzylidene acetophenone)} substituted phenothiazine $4 \mathbf{j}$

Yield $77 \%, \quad$ M.P $227^{\circ} \mathrm{C}$ : IR(KBr); $3540(\mathrm{NH}-$ pyrrole), 34629 (NH-phenothiazine) , 3354 (NHpyrazole) $1635(\mathrm{ArH}), 1465(\mathrm{C}=\mathrm{N}), 811(\mathrm{C}-\mathrm{N})$, 740(C-S); ${ }^{1} \mathrm{HNMR}$ (300 MHz DMSO) $\delta 8.28$ $(1 \mathrm{H}, \mathrm{s}, \mathrm{NH}-$ pyrrole $) \quad, \quad 7.8 \quad(1 \mathrm{H}, \mathrm{s}, \mathrm{N}-\mathrm{H}-$ phenothiazine), $7.28(1 \mathrm{H}, \mathrm{s}, \mathrm{NH}-$ pyrazole) $6.8(5 \mathrm{H}$, $\mathrm{m}, \mathrm{ArH})$.

\section{Conclusion:}

During our synthesis, we have used microwave methodology for the synthesis of 7 (4-Arylidene benzylidene acetophenone) substituted phenothiazines. Microwave assisted organic synthesis have fascinated the chemist due to its usefulness with reduction of reaction time, environmental friendly methodology etc. compound (4b, 4d, 4f, 4j) was effective against E.Coli, S.aureus B. substillis C. albicans A. niger, compounds $(4 \mathrm{a}, 4 \mathrm{c}, 4 \mathrm{e}, 4 \mathrm{~g}, 4 \mathrm{i})$ effective against C. albicans A. niger

Table I- Antibacterial and antifungal activities of compounds $4 a-j$.

\begin{tabular}{|c|c|c|c|c|c|}
\hline \multicolumn{3}{|c|}{ Antibacterial activity } & \multicolumn{2}{|c|}{ Antifungal activity } & \multirow[b]{2}{*}{ A. niger } \\
\hline Compc & d S.aureus & B. substillis & E. coli & C. albicans & \\
\hline $4 a$ & ++ & ++ & + & ++ & +++ \\
\hline $4 b$ & ++ & ++ & +++ & + & ++ \\
\hline $4 c$ & +++ & ++ & +++ & - & ++ \\
\hline $4 d$ & ++ & +++ & ++ & ++ & +++ \\
\hline $4 \mathrm{e}$ & + & ++ & + & + & + \\
\hline $4 \mathrm{f}$ & +++ & + & +++ & +++ & - \\
\hline $4 g$ & +++ & ++ & +++ & +++ & ++ \\
\hline $4 \mathrm{~h}$ & ++ & +++ & + & + & ++ \\
\hline $4 i$ & +++ & ++ & +++ & ++ & + \\
\hline $4 j$ & ++ & + & ++ & + & +++ \\
\hline SM & +++ & +++ & ++++ & ++++ & +++ \\
\hline GF & & & & & \\
\hline
\end{tabular}

SM (Streptomycin) and GF (Griesofulvin). The inhibition diameter in

Mm: $(-)<6,(+) 7-9,(++) 10-15,(+++) 16-22,(++++) 23-28$.

Table. 2-comparative study data of compounds $4 a-j$

\begin{tabular}{|c|c|c|c|c|c|c|c|}
\hline \multirow[b]{2}{*}{ Compd } & \multirow[b]{2}{*}{ M.P. $\left({ }^{\circ} \mathrm{C}\right)$} & \multicolumn{3}{|c|}{ Reaction time } & \multicolumn{3}{|c|}{ Yield (\%) } \\
\hline & & $\begin{array}{l}\text { Microwave } \\
\text { solid } \\
\text { phase } \\
\text { (min) }\end{array}$ & $\begin{array}{l}\text { Solvent } \\
\text { phase } \\
\text { (min) }\end{array}$ & $\begin{array}{l}\text { Classical } \\
\text { (hr) }\end{array}$ & $\begin{array}{l}\text { Solid } \\
\text { phase }\end{array}$ & $\begin{array}{l}\text { Microwave } \\
\text { Solvent } \\
\text { phase }\end{array}$ & Classical \\
\hline $4 a$ & $216^{0}$ & 5 & 6 & 8 & 77 & 72 & 71 \\
\hline $4 \mathrm{~b}$ & $207^{0}$ & 6 & 6 & 8 & 82 & 80 & 67 \\
\hline $4 c$ & $121^{0}$ & 5.5 & 6.5 & 7 & 78 & 73 & 65 \\
\hline $4 d$ & $210^{0}$ & 6 & 6 & 8 & 85 & 83 & 58 \\
\hline $4 \mathrm{e}$ & 1940 & 5 & 7 & 7 & 79 & 78 & 78 \\
\hline $4 \mathrm{f}$ & $215^{0}$ & 6 & 6 & 8 & 80 & 78 & 66 \\
\hline $4 g$ & $199^{\circ}$ & 5 & 7 & 7 & 85 & 76 & 64 \\
\hline $4 \mathrm{~h}$ & $220^{\circ}$ & 5.5 & 6 & 8 & 83 & 84 & 81 \\
\hline $4 i$ & $123^{0}$ & 6 & 7 & 7 & 68 & 62 & 66 \\
\hline $4 j$ & $227^{0}$ & 5 & 7 & 7 & 69 & 64 & 77 \\
\hline
\end{tabular}


Table. 3- Characterization data of Newly synthesized compounds (4a-j)

\begin{tabular}{|c|c|c|c|c|c|c|c|c|}
\hline \multirow[t]{2}{*}{ Comp } & \multirow[t]{2}{*}{$\mathrm{R}$} & \multirow[t]{2}{*}{ Mol Formula } & \multirow[t]{2}{*}{$\begin{array}{l}\text { M.P. } \\
\left({ }^{\circ} \mathrm{C}\right)\end{array}$} & \multirow[t]{2}{*}{$\begin{array}{l}\text { Yield } \\
(\%)\end{array}$} & \multicolumn{4}{|c|}{$\begin{array}{l}\text { Analysis formula } \\
\text { (calcd)\% (obs) }\end{array}$} \\
\hline & & & & & $\mathrm{C}$ & $\mathrm{H}$ & $\mathrm{N}$ & $\mathrm{S}$ \\
\hline $4 a$ & $-\mathrm{H}$ & C19H13 ON30S & 216 & 71 & $\begin{array}{l}69.0 \\
(69.1)\end{array}$ & $\begin{array}{l}4.2 \\
(4.3)\end{array}$ & $\begin{array}{l}16.9 \\
(16.7)\end{array}$ & \begin{tabular}{|l|}
9.7 \\
$(9.4)$
\end{tabular} \\
\hline $4 \mathrm{~b}$ & $2-\mathrm{OH}$ & C19H13O2N3S & 207 & 67 & $\begin{array}{l}65.76 \\
(65.74)\end{array}$ & $\begin{array}{l}4.0 \\
(4.0) \\
\end{array}$ & $\begin{array}{l}16.1 \\
(16.2)\end{array}$ & \begin{tabular}{|l|}
9.2 \\
$(9.1)$ \\
\end{tabular} \\
\hline $4 c$ & $3-\mathrm{OH}$ & C19H13O2N3S & 121 & 65 & $\begin{array}{l}65.76 \\
(65.74) \\
\end{array}$ & \begin{tabular}{|l|}
4.0 \\
$(4.0)$ \\
\end{tabular} & $\begin{array}{l}16.1 \\
(16.2)\end{array}$ & \begin{tabular}{|l|}
9.2 \\
$(9.1)$ \\
\end{tabular} \\
\hline $4 d$ & $4-\mathrm{OH}$ & C19H13O2N3S & 210 & 58 & $\begin{array}{l}65.76 \\
(65.74) \\
\end{array}$ & \begin{tabular}{|l|}
4.0 \\
$(4.0)$ \\
\end{tabular} & $\begin{array}{l}16.1 \\
(16.2) \\
\end{array}$ & \begin{tabular}{|l|}
9.2 \\
$(9.1)$ \\
\end{tabular} \\
\hline $4 \mathrm{e}$ & $2-\mathrm{NO} 2$ & C19H12O3N4S & 194 & 78 & $\begin{array}{l}60.7 \\
(60.6)\end{array}$ & $\begin{array}{l}3.4 \\
(3.6)\end{array}$ & $\begin{array}{l}18.1 \\
(18.2)\end{array}$ & \begin{tabular}{|l|}
8.5 \\
$(8.4)$
\end{tabular} \\
\hline $4 \mathrm{f}$ & $3-\mathrm{NO} 2$ & C19H12O3N4S & 215 & 68 & $\begin{array}{l}60.7 \\
(60.6)\end{array}$ & $\begin{array}{l}3.4 \\
(3.6) \\
\end{array}$ & $\begin{array}{l}18.1 \\
(18.2)\end{array}$ & \begin{tabular}{|l|}
8.5 \\
$(8.4)$ \\
\end{tabular} \\
\hline $4 g$ & $4-\mathrm{NO} 2$ & C19H12O3N4S & 199 & 64 & $\begin{array}{l}60.7 \\
(60.6)\end{array}$ & $\begin{array}{l}3.4 \\
(3.6) \\
\end{array}$ & $\begin{array}{l}18.1 \\
(18.2) \\
\end{array}$ & \begin{tabular}{|l|}
8.5 \\
$(8.4)$ \\
\end{tabular} \\
\hline $4 \mathrm{~h}$ & $\mathrm{~N}(\mathrm{CH} 3) 21$ & $\mathrm{C} 21 \mathrm{H} 18 \mathrm{ON} 4 \mathrm{~S}$ & 220 & 81 & $\begin{array}{l}67.5 \\
(67.6)\end{array}$ & $\begin{array}{l}5.3 \\
(5.1)\end{array}$ & $\begin{array}{l}18.7 \\
(18.5)\end{array}$ & \begin{tabular}{|l|}
8.5 \\
$(8.3)$ \\
\end{tabular} \\
\hline $4 i$ & $-\mathrm{Cl}$ & $\mathrm{C} 19 \mathrm{H} 12 \mathrm{ON} 3 \mathrm{ClS}$ & 123 & 66 & $\begin{array}{l}62.5 \\
(62.6)\end{array}$ & \begin{tabular}{|l|}
5.3 \\
$(5.3)$ \\
\end{tabular} & $\begin{array}{l}15.3 \\
(15.2) \\
\end{array}$ & \begin{tabular}{|l|}
8.7 \\
$(8.8)$ \\
\end{tabular} \\
\hline $4 j$ & $-\mathrm{OCH} 3$ & $\mathrm{C} 2 \mathrm{OH} 15 \mathrm{O} 2 \mathrm{~N} 3 \mathrm{~S}$ & 227 & 77 & $\begin{array}{l}66.5 \\
(66.6) \\
\end{array}$ & $\begin{array}{l}4.3 \\
(4.3) \\
\end{array}$ & $\begin{array}{l}15.5 \\
(15.4) \\
\end{array}$ & \begin{tabular}{|l|}
8.9 \\
$(8.6)$ \\
\end{tabular} \\
\hline
\end{tabular}

\section{Acknowledgement}

The authors extend his heartful thanks to principal, BDCOE, wardha for providing the basic facilities for the present investigation. Thanks are also due to Dr. (Mrs.). J. S. meshram, Department of Chemistry, RTM Nagpur University, Nagpur for providing data and facilities of microwave oven without which the present experimental work could not be completed.

\section{References}

1. S. Ishida, A. Matsuda and A. Kawamura, Chemotherapy, 8, 146(1960); Chem. Abstr., $54,22844 c\left(1^{-} 960\right.$.

2. K. J. Mehta, V. S. Patel and A. R. Parikh, J. Indian Chem. Soc., 50, 241(1978).

3. V. Mudaliar and V. Joshi, Indian J. Chem., 34B, 456(1995).

4. G. Hosni and S. P. Saad, Acta Chim. Acad. Sci. Hung., 86, 263(1995); Chem. Abustr., 84, 30959w(1976).

5. O. H. Hishmat, H. I. EI-Diwani and F. R. Melek, Indian J. Chem, 35B, 30(1996).

9. H. J. McQuay et. al., Clin. Pharmacol.

Ther. 39, 1986, 89.

10. Buu-Hoi et. al., J. Med. Pharm. Chem. 1, 1959, 23.

11. Janssen et. al., J. Am. Chem. Soc, 73, 1951, 4040.
12. J. W. Corcoran, F. E. Hahn, Hahn in Antibiotics vol. 3, Eds. (Springer-verlag, New York, 1975, 79-100.

13. Harris et. al., J. Med. Che, 8, 1965, 478.

14. S. Wang et. al., J. Pharmacol. Exp. Ther. 185, 1973, 127.

15. Tolman et. al., J. Am. Chem. Soc. 91, 1969, 2102.

16. Della Bella, Boll.Chim Farm 11, 1972, 519.

17. C. E. Steele, W. L. Jefferson, Curr. Med. Res. Opin, 8, 382, 1983.

18. Kostanecki S Vand Tambor, Ber, 32, 1899, 1921

19. Wilson C W, J Am Chem Soc, 1939, 2303. 20. Schrauffstatter E and Deutsch S, Chem Abstr, 44, 1950, 3568c.

21.Adityachaudhury N, Kirtaniya C L and Mukherjee B, Tetraheadron, 27, 1971, 2111.

22. Hansel R and Sauer H, Planta Madica, 15, $1967,443$.

23. Cardillo G, Merlini L, Nasini G and Salvadori P, J Chem Soc (C), 197 1, 3967.

24. Furuya $\mathrm{T}$, Matsumoto $\mathrm{K}$ and Hikichi $\mathrm{M}$, Tetrahedron Letters, 1971, 2561.

25. Seikel M K and Geissman T A, J Am Chem Soc, 72, 1950, 5720 . 